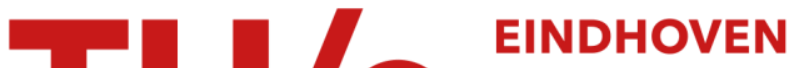 \\ UNIVERSITY OF \\ TECHNOLOGY
}

\section{Wireless network control of interacting Rydberg atoms}

\section{Citation for published version (APA):}

Sanders, J., Bijnen, van, R. M. W., Vredenbregt, E. J. D., \& Kokkelmans, S. J. J. M. F. (2014). Wireless network control of interacting Rydberg atoms. Physical Review Letters, 112(16), 1-5. [163001].

https://doi.org/10.1103/PhysRevLett.112.163001

DOI:

10.1103/PhysRevLett.112.163001

Document status and date:

Published: 01/01/2014

\section{Document Version:}

Publisher's PDF, also known as Version of Record (includes final page, issue and volume numbers)

\section{Please check the document version of this publication:}

- A submitted manuscript is the version of the article upon submission and before peer-review. There can be important differences between the submitted version and the official published version of record. People interested in the research are advised to contact the author for the final version of the publication, or visit the $\mathrm{DOI}$ to the publisher's website.

- The final author version and the galley proof are versions of the publication after peer review.

- The final published version features the final layout of the paper including the volume, issue and page numbers.

Link to publication

\section{General rights}

Copyright and moral rights for the publications made accessible in the public portal are retained by the authors and/or other copyright owners and it is a condition of accessing publications that users recognise and abide by the legal requirements associated with these rights.

- Users may download and print one copy of any publication from the public portal for the purpose of private study or research.

- You may not further distribute the material or use it for any profit-making activity or commercial gain

- You may freely distribute the URL identifying the publication in the public portal.

If the publication is distributed under the terms of Article 25fa of the Dutch Copyright Act, indicated by the "Taverne" license above, please follow below link for the End User Agreement:

www.tue.nl/taverne

Take down policy

If you believe that this document breaches copyright please contact us at:

openaccess@tue.nl

providing details and we will investigate your claim. 


\title{
Wireless Network Control of Interacting Rydberg Atoms
}

\author{
Jaron Sanders, ${ }^{*}$ Rick van Bijnen, ${ }^{\dagger}$ Edgar Vredenbregt, and Servaas Kokkelmans \\ Eindhoven University of Technology, P.O. Box 513, 5600 MB Eindhoven, The Netherlands
}

(Received 12 December 2013; published 22 April 2014)

\begin{abstract}
We identify a relation between the dynamics of ultracold Rydberg gases in which atoms experience a strong dipole blockade and spontaneous emission, and a stochastic process that models certain wireless random-access networks. We then transfer insights and techniques initially developed for these wireless networks to the realm of Rydberg gases, and explain how the Rydberg gas can be driven into crystal formations using our understanding of wireless networks. Finally, we propose a method to determine Rabi frequencies (laser intensities) such that particles in the Rydberg gas are excited with specified target excitation probabilities, providing control over mixed-state populations.
\end{abstract}

DOI: 10.1103/PhysRevLett.112.163001

PACS numbers: 32.80.Rm, 02.50.Ga, 89.20.Ff

Stochastic processes play a ubiquitous role in interacting particle systems. Glauber initiated a study of the stochastic Ising model in 1963 [1], and similar models are actively investigated in probability theory, often applied to very different systems [2,3]. The two seemingly disparate interacting particle systems we study in this Letter, are a gas of ultracold Rydberg atoms [4] accompanied by a dissipative mechanism, and a wireless random-access network, made up of for example electronic transmitters in communication networks [5]. It turns out that their dynamics can be described, under certain conditions, with the same equations. Indeed, Rydberg atoms exhibit a strong interaction, while simultaneously active transmitters would lead to interference at receivers, both resulting in complicated large-scale system behavior.

Rydberg gases consist of atoms that can be in either a ground state or an excited state with a high principal quantum number. When an atom is excited, the energy levels of neighboring atoms shift. This makes it unlikely for neighboring atoms to also excite, and we call this effect the dipole blockade [6,7]. The dipole blockade is at the basis of quantum information and quantum gate protocols $[6,8,9]$, and also allows for a phase transition to ordered structures [10]. Experimentally, the CNOT gate has been demonstrated [9], while also the first ordered Rydberg structures have been observed [11]. Recent experiments are geared towards leveraging the dipole blockade to create Rydberg crystals, i.e. formations of regularly spaced excited atoms. A proposed method is to use chirped laser pules [12-14], and another utilizes a dissipation mechanism, specifically spontaneous emission [15].

Nowadays, transmitters in wireless networks share a transmission medium through the use of distributed random-access protocols. We focus on wireless networks operating according to the CSMA protocol [16], which lets transmitters autonomously decide when to start a transmission based on the level of activity in their environment, usually estimated through measurements of interference and signal-to-noise ratios. If too many neighbors are sensed to be transmitting, the transmitter postpones its activation and tries again at a random later point in time. We see that transmitters experience blocking effects similar to the Rydberg dipole blockade, which sparked our original interest to compare their mathematical models [17]. Mathematical models of wireless networks were already being studied because of our increasing demands on our communication infrastructures, and we focussed our attention on stochastic models of CSMA that were originally considered in $[5,18,19]$.

This Letter uses the fact that rate equations adequately describe the Rydberg gas when spontaneous emission is introduced to the model [20], and we interpret the rate equations as Kolmogorov forward equations [21] that describe the transient evolution of a stochastic model reminiscent of CSMA.

Regarding the Rydberg gas, we consider a gas of $N$ atoms in the $\mu$-Kelvin regime, to which we apply the frozen gas approximation by neglecting the kinetic energy of the system. The atoms are thus considered fixed at positions $\boldsymbol{r}_{i} \in \mathbb{R}^{3}$ for $i=1, \ldots, N$. The ultracold atoms are subjected to two lasers with associated Rabi frequencies $\Omega_{\mathrm{e}}, \Omega_{\mathrm{r}}$, respectively, that facilitate excitation from the ground state $|g\rangle$ to an intermediate state $|e\rangle$, and from the intermediate state $|e\rangle$ to a Rydberg state $|r\rangle$. We also assume that the intermediate state decays with rate $\Gamma$, through spontaneous emission. In principle, detuning of the laser frequencies could be taken into account, but here we leave it out for simplicity.

The system description of a wireless random-access network is similar to that of the Rydberg gas, but with different terminology. A wireless random-access network can be modeled as consisting of $N$ transmitter-receiver pairs, and each transmitter can be either active (1) or nonactive (0). When active, a transmitter transmits data for an exponentially distributed time with mean $1 / \mu$. Similarly, a nonactive transmitter repeatedly attempts to become active after exponentially distributed times with mean 
$1 / \nu$. Figure 1 summarizes our modeling assumptions thus far.

For a single atom, we can write down the optical Bloch equations. As in [20], we then conclude that if (i) the upper transition is much more weakly driven than the lower one $\left(\Omega_{\mathrm{r}} \ll \Omega_{\mathrm{e}}\right)$, and (ii) the decay rate of the intermediate level is much larger than the Rabi frequency driving between $|e\rangle$ and $|r\rangle\left(\Omega_{\mathrm{r}} \ll \Gamma\right)$, that then the excitation dynamics are described using the rate equation,

$$
\frac{d p_{1}(t)}{d t}=\nu p_{0}(t)-\mu p_{1}(t) .
$$

Here, $p_{0}(t)$ and $p_{1}(t)$ denote the probabilities that the atom is (effectively) in the ground state or the Rydberg state, respectively. Furthermore,

$$
\mu=\frac{2 \Gamma \Omega_{\mathrm{r}}^{4}}{\left(\Omega_{\mathrm{r}}^{2}-2 \Omega_{\mathrm{e}}^{2}\right)^{2}+2 \Gamma^{2}\left(\Omega_{\mathrm{e}}^{2}+\Omega_{\mathrm{r}}^{2}\right)}, \quad \text { and } \quad \nu=\frac{\Omega_{\mathrm{e}}^{2}}{\Omega_{\mathrm{r}}^{2}} \mu
$$

denote the transition rates between the ground and Rydberg state. It is noteworthy that Eq. (1) also describes the time evolution of a single, noninteracting transmitter. The $p_{0}(t)$ and $p_{1}(t)$ are then the probabilities that the transmitter is nonactive or active, respectively.

When dealing with many-particle systems, however, we have to take particle interactions into account. The atoms in Rydberg gases, and the transmitters in wireless networks, interact with each other. Specifically, if an atom is in the Rydberg state, other nearby atoms experience a dipole blockade [14]. Transmitters that detect high levels of interference and low signal-to-noise ratios (because of their neighbors) postpone their activation.

We will model the dipole blockade, as well as the interference constraints on transmitters, using a unit-disk interference model. The unit-disk interference model involves the assumption that atoms (transmitters) within a distance $R$ of each other cannot simultaneously be in the Rydberg state (active). For Rydberg gases, this assumption is in line with measurements and simulations of pair correlation functions between atoms in the Rydberg state, which show a sharp cutoff when plotted as a function of the distance between the atoms $[11,22]$. The collection of possible configurations is thus

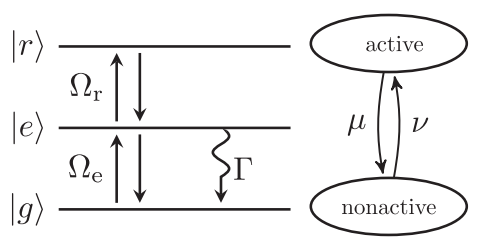

FIG. 1. (left) An atom can transition between a ground, intermediate and a Rydberg state. (right) A transmitter can change between nonactive and active.

$$
\mathcal{S}=\left\{\boldsymbol{\sigma} \in\{0,1\}^{N} \mid d\left(\boldsymbol{r}_{i}, \boldsymbol{r}_{j}\right)>R \forall_{i \neq j: \sigma_{i}=\sigma_{j}=1}\right\},
$$

and these configurations $\boldsymbol{\sigma}=\left(\sigma_{1}, \ldots, \sigma_{N}\right)^{\mathrm{T}}$ will be called feasible. The notation is such that if $\sigma_{i}=0$ or 1 , atom $i$ is in the ground or Rydberg state, respectively. Similarly, $\sigma_{i}=0$ or 1 if transmitter $i$ is nonactive or active, respectively.

There are certainly practical differences between Rydberg gases and wireless networks. In wireless networks, every transmitter can have its own activation $\left(\nu_{i}\right)$ and deactivation rate $\left(\mu_{i}\right)$. To achieve the same effect in Rydberg gases, we will assume that the two-step laser can be split into $M \ll N$ spots with radius $S$, and that each spot $i=1, \ldots, M$ has a different laser intensity $E_{i}$. Each laser spot contains a cluster of atoms, and with this setup, the atoms within each cluster may be subjected to a different Rabi frequency. We assume that $S \ll R$, so that we can treat each spot as being synonymous to one atom, and we will replace the symbol $M$ by $N$ for notational convenience. Each atom (spot) $i=1, \ldots, N$ will thus experience its own transition rates $\nu_{i}, \mu_{i}$. Figure 2 summarizes the blockade effect, our assumptions on the laser spots, and the unit-disk interference model.

For both models, the probability of observing the system in state $\sigma \in \mathcal{S}$ at time $t$, denoted by $p_{\boldsymbol{\sigma}}(t)$, is described by the master equation

$$
\frac{d p_{\sigma}(t)}{d t}=\sum_{\eta \in \mathcal{S}} Q_{\sigma, \eta} p_{\eta}(t)
$$

where $Q$ denotes a transition rate matrix. The master equation Eq. (4) can be interpreted as a Kolmogorov forward equation, which characterizes a Markov process [21,23].

The off-diagonal elements of $Q \in \mathbb{R}^{|\mathcal{S}| \times|\mathcal{S}|}$ describe the dynamics of this stochastic process. Denoting the $N$-dimensional vector with a one in the $i$ th position by $\boldsymbol{e}_{i}$, we have that when the system is in state $\boldsymbol{\sigma} \in \mathcal{S}$, it jumps to states $\boldsymbol{\sigma}+\boldsymbol{e}_{i}, i=1, \ldots, N$, with rate $Q_{\sigma, \sigma+\boldsymbol{e}_{i}}=\nu_{i}$ if $\boldsymbol{\sigma}+\boldsymbol{e}_{i} \in \mathcal{S}$, and to states $\boldsymbol{\sigma}-\boldsymbol{e}_{i}, i=1, \ldots, N$, with rate

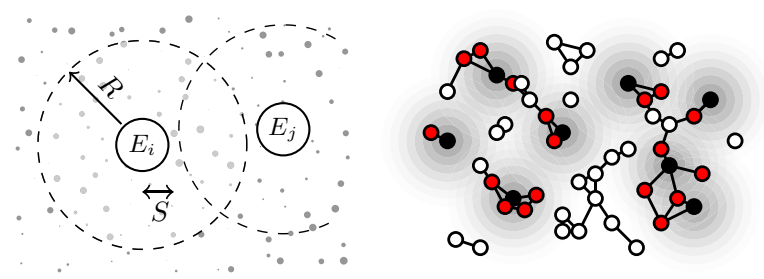

FIG. 2 (color online). The Rydberg blockade prevents atoms within a radius $R$ from becoming Rydberg atoms (left). This interaction can be described using an interference graph where edges indicate which neighboring particles would block each other, which is part of the wireless network model (right). Active transmitters (black) prevent neighboring transmitters (red) from becoming active. Non-neighboring nonactive transmitters (white) can become active. 
$Q_{\boldsymbol{\sigma}, \boldsymbol{\sigma}-\boldsymbol{e}_{i}}=\mu_{i}$ if $\boldsymbol{\sigma}-\boldsymbol{e}_{i} \in \mathcal{S}$. All other off-diagonal elements of $Q$ are set to zero, which (for the Rydberg gas model) means that we neglect multiphoton processes. For completeness, we note that the diagonal elements are given by $Q_{\sigma, \sigma}=-\sum_{\eta \neq \sigma} Q_{\sigma, \eta}$. We conclude that the stochastic process described by the generator matrix $Q$, which we denote by $\{\boldsymbol{X}(t)\}_{t \geq 0}$, is a model for wireless random-access networks, as well as Rydberg gases.

We now investigate steady states of the Rydberg gas, using our understanding of wireless networks. The equilibrium fraction of time that the system spends in state $\sigma$ is given by

$$
\pi_{\boldsymbol{\sigma}}(\boldsymbol{\nu}, \boldsymbol{\mu})=\frac{1}{Z(\boldsymbol{\nu}, \boldsymbol{\mu})} \prod_{i=1}^{N}\left(\frac{\nu_{i}}{\mu_{i}}\right)^{\sigma_{i}}, \quad \boldsymbol{\sigma} \in \mathcal{S},
$$

with $Z(\boldsymbol{\nu}, \boldsymbol{\mu})$ denoting the normalization constant. The equilibrium distribution depends solely on the ratios $\nu_{i} / \mu_{i}$, and proving that it is in fact the equilibrium distribution can be done by observing that it satisfies the detailed balance equations [23], $\pi_{\sigma} Q_{\sigma, \eta}=\pi_{\eta} Q_{\eta, \sigma}$, for all $\sigma, \eta \in \mathcal{S}$.

Consider the special case in which all particles make their transition at the same rate, and set $\nu_{i}=\nu$ and $\mu_{i}=\mu$ for $i=1, \ldots, N$ accordingly. When $\nu / \mu \rightarrow \infty$, the equilibrium probability of observing the system in state $\sigma \in \mathcal{S}$ converges to

$$
\pi_{\sigma}(\boldsymbol{\nu}, \boldsymbol{\mu})=\frac{1}{Z(\boldsymbol{\nu}, \boldsymbol{\mu})}\left(\frac{\nu}{\mu}\right)^{\sum_{i=1}^{N} \sigma_{i}} \rightarrow \frac{\mathbb{1}[\boldsymbol{\sigma} \in \mathcal{I}]}{|\mathcal{I}|},
$$

where $\mathcal{I}$ denotes the collection of maximum independent sets of $\mathcal{S}$. In the present context, a maximum independent set is a configuration in which the largest number of particles are active, i.e., in the Rydberg state. We call these configurations dominant, because the probability of observing a dominant configuration $\sigma \in \mathcal{I}, \pi_{\sigma}$, is large compared to (i) the probability of observing a configuration $\boldsymbol{\eta} \notin \mathcal{I}, \pi_{\eta}$, when $\nu \gg \mu$, and (ii) the probability of observing any but the dominant configuration, $\sum_{\eta \notin \mathcal{I}} \pi_{\eta}$, when $\nu \gg N \mu$.

The active particles in dominant configurations typically form patterns, which resemble crystal structures. Consider for instance an $n \times m$ lattice of particles exhibiting nearestneighbor blocking, where $n, m \in \mathbb{N}^{+}$. For such networks, the active particles in the dominant configuration follow a checkerboard pattern, as illustrated in Fig. 3. When both $n$ and $m$ are even, two dominant configurations exist, which we henceforth refer to as the even and odd configuration.

Our analysis reveals that when $\Omega_{\mathrm{r}} \ll \Omega_{\mathrm{e}}$, the Rydberg gas spends more time in a dominant configuration than in another configuration. The time it takes for the system to switch between different dominant configurations is related to the mixing time of the system, i.e. the time required for the

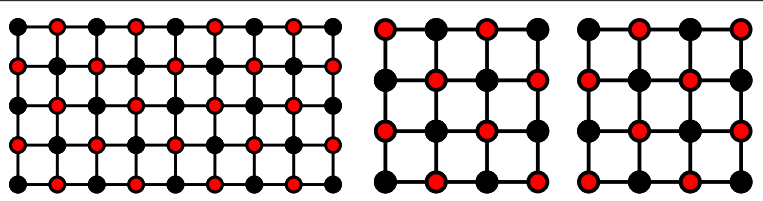

FIG. 3 (color online). Dominant configurations in a (left) $9 \times 5$ lattice and (middle, right) $4 \times 4$ lattice with nearest-neighbor blocking.

Markov process to get sufficiently close to stationarity [24]. Depending on the topology, the mixing time can be large when $\Omega_{\mathrm{r}} \ll \Omega_{\mathrm{e}}$, implying that once the system is in a dominant configuration, it tends to stay there for a long time. It is noteworthy that simulations of a driven dissipative Rydberg gas confirmed the formation of crystalline structures in $[15,25,26]$, and here we have explained how such formations appear using our connection to wireless networks.

We are also able to investigate the time $\tau$ it takes until the process reaches a dominant configuration. The hitting time $\tau$ of the dominant configuration is the first moment at which the system reaches the even or odd dominant configuration. The random variable $\tau$ is of interest, because it is a measure for how long the experimentalist has to wait before a dominant configuration has appeared.

To illustrate this, we have simulated sample paths of $\boldsymbol{X}(t)$ on even $n \times n$ lattice topologies. Histograms of the hitting time distributions for grids of several sizes are shown in Fig. 4, as well as the normalized average number of excited particles. Note that the average hitting time increases as lattices become larger.

We now describe a wireless network algorithm in the context of Rydberg gases, to determine Rabi frequencies (laser intensities) such that particles in the Rydberg gas are excited with specified target excitation probabilities. The algorithm was developed in [27] to achieve maximum throughput in wireless networks in a distributed fashion, and was later generalized for implementation in productform networks [28]. In Supplemental Material [29], we provide a short discussion of the algorithm in its original context, and we explain that the algorithm is solving an inversion problem that can be NP hard.

The wireless network algorithm can be applied to the Rydberg atoms by iteratively setting
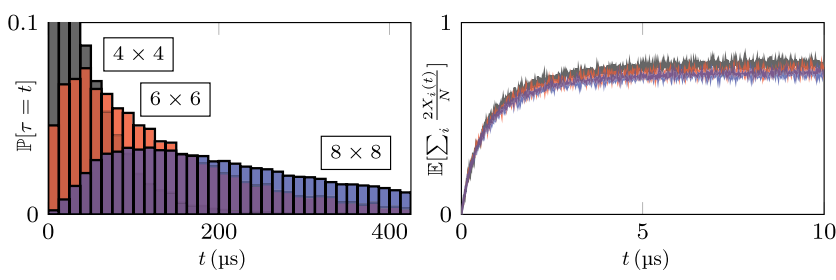

FIG. 4 (color online). Histograms of the hitting time distributions (left), and normalized average number of excited particles (right) for lattices of sizes $4 \times 4,6 \times 6$, and $8 \times 8$. Here, $\Gamma=2 \pi \times 6 \mathrm{MHz}, \Omega_{\mathrm{e}, i}=2 \pi \times 3 \mathrm{MHz}$ and $\Omega_{\mathrm{r}, i}=2 \pi \times 1 \mathrm{MHz}$. 


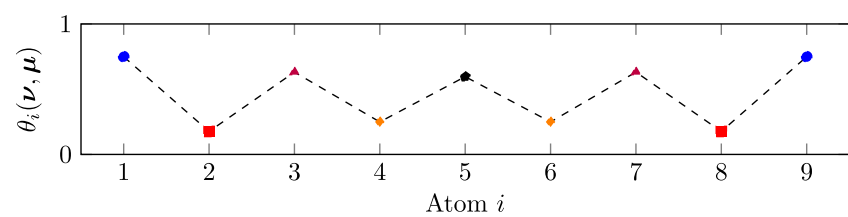

FIG. 5 (color online). The $\theta_{i}(\boldsymbol{\nu}, \boldsymbol{\mu})$ for $N=9, b=1$, and $\nu_{i} / \mu_{i}=10$.

$$
\left|\Omega_{e, i}^{[n+1]}\right|=\left|\Omega_{\mathrm{e}, i}^{[n]}\right| \exp \left(-\frac{1}{2} a^{[n+1]}\left(\hat{\theta}_{i}^{[n+1]}-\phi_{i}\right)\right),
$$

for atoms $i=1, \ldots, N$. Here, $n \in \mathbb{N}^{+}$indexes each iteration, and the $a^{[n]}$ denote algorithm step sizes that are typically chosen as a decreasing sequence. The $\hat{\theta}_{i}^{[n+1]}$ denote empirically obtained estimates of the probabilities of observing atom $i=1, \ldots, N$ in the Rydberg state, $\theta_{i}$, and $\phi_{i}$ denotes the target probability of observing atom $i$ in the Rydberg state. The algorithm in Eq. (7) seeks $\boldsymbol{\Omega}_{\mathrm{e}}^{\mathrm{opt}}$ such that $\boldsymbol{\theta}\left(\boldsymbol{\Omega}_{\mathrm{e}}^{\mathrm{opt}}, \boldsymbol{\Omega}_{\mathrm{r}}\right)=\boldsymbol{\phi}$.

In wireless networks, an estimate $\hat{\theta}_{i}$ can be obtained through online observation of a transmitter's activity [Supplemental Material [29], Eq. (1)]. Experimentally observing the evolution of a particle system through time however is difficult. Instead, we can (i) determine an estimate $\hat{\boldsymbol{\theta}}$ of $\boldsymbol{\theta}$ using simulation, or (ii) use repeated experimentation to determine an estimate $\hat{\boldsymbol{\theta}}$ of $\boldsymbol{\theta}$. With the latter approach, we forego our mathematical guarantee of convergence, but the design principles that guaranteed the convergence in the former method still hold. That is, we need to improve the quality of $\hat{\boldsymbol{\theta}}$ as the number of iterations $n$ increases.

For every $n$th iteration of the algorithm, we can for example reinitialize the process $m^{[n]}$ times and determine the state the process is in at some time $T^{[n]}$. Denoting these samples by $X_{i}^{[n, s]}\left(T^{[n]}\right)$, with $n \in \mathbb{N}^{+}$and $s \in\left\{1, \ldots, m^{[n]}\right\}$, we can calculate

$$
\hat{\theta}_{i}^{[n]}=\frac{1}{m^{[n]}} \sum_{s=1}^{m^{[n]}} \mathbb{1}\left[X_{i}^{[n, s]}\left(T^{[n]}\right)=1\right], \quad i=1, \ldots, N,
$$

which, for sufficiently large $T^{[n]}$ and $m^{[n]}$, provides an estimate of the equilibrium probability that particle $i$ is in the Rydberg state. Intuitively, we expect that $T^{[n]}$ should be at least of the order of the mixing time (that is to say, the system should be close to equilibrium).

As an example, we focus on a system of $i=1, \ldots, N$ atoms positioned on a line, that block the first $b$ neighbors on both sides. We consider the problem of determining $\boldsymbol{\Omega}_{\mathrm{e}}$ such that each atom is excited with equal probability $\phi \in(0,1)$. This problem is nontrivial because the atoms at the border have fewer neighbors that block them and are therefore excited with higher probability. Moreover, this effect propagates through the system, which can be verified by an analytical evaluation of the probabilities of observing atom $i$ in the Rydberg state,

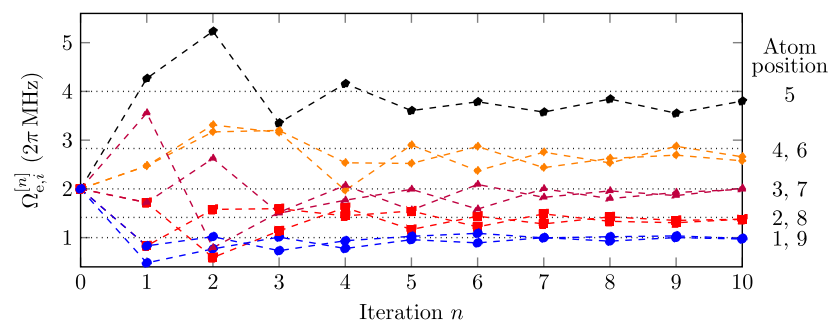

FIG. 6 (color online). Algorithm output when $N=9, b=4$, $\Gamma=2 \pi \times 6 \mathrm{MHz}$, and $\Omega_{\mathrm{r}, i}=2 \pi \times 1 \mathrm{MHz}$. The dotted lines indicate $\boldsymbol{\Omega}_{\mathrm{e}}^{\mathrm{opt}}$.

$$
\theta_{i}(\boldsymbol{\nu}, \boldsymbol{\mu})=\sum_{\sigma \in \mathcal{S}} \sigma_{i} \pi_{\sigma}(\boldsymbol{\nu}, \boldsymbol{\mu}), \quad i=1, \ldots, N
$$

as shown in Fig. 5.

We consider this particular example because we can again utilize our connection to wireless networks and provide an analytical expression for $\boldsymbol{\Omega}_{\mathrm{e}}^{\mathrm{opt}}$. As shown in [30], we need to set

$$
\left(\frac{\Omega_{\mathrm{e}, i}^{\mathrm{opt}}}{\Omega_{\mathrm{r}, i}}\right)^{2}=\frac{\phi}{1-(1+b) \phi}\left(\frac{1-b \phi}{1-(1+b) \phi}\right)^{w(i)-w(1)}
$$

for $i=1, \ldots, N$, in order to have $\theta_{i}\left(\boldsymbol{\Omega}_{\mathrm{e}}^{\mathrm{opt}}, \boldsymbol{\Omega}_{\mathrm{r}}\right)=\phi$ for $i=1, \ldots, N$. Here, $w(i)=\min \{i+b, N\}-\max \{1, i-b\}$ denotes the number of other atoms that atom $i$ blocks if it is excited.

In order to illustrate the algorithm applied to this system, we utilize the following simulation procedure. We repeatedly simulate the Rydberg gas by generating sample paths $\boldsymbol{X}^{[n, s]}(t)$ using the generator matrix in Eq. (4). Subsequently, we calculate an estimate of the excitation probabilities through Eq. (8), and update the Rabi frequencies according to the algorithm in Eq. (7). In every $n$th iteration of our algorithm, we set the maximum simulation time to $T^{[n]}=250 \mu \mathrm{s}$, produce $m^{[n]}=25 n^{2}$ samples, and choose step size $a^{[n]}=100 /(10+\sqrt{n})$. The target excitation probability of the algorithm is set to $\phi=1 / 6$. The resulting Rabi frequencies are shown in Fig. 6 and approach the exact solution given by Eq. (10), $\boldsymbol{\Omega}_{\mathrm{e}}^{\mathrm{opt}}=(1, \sqrt{2}, 2,2 \sqrt{2}, 4,2 \sqrt{2}, 2, \sqrt{2}, 1)^{\mathrm{T}} \cdot 2 \pi \mathrm{MHz}$. The excitation probabilities approach the target $\phi$, which can be verified by evaluating Eq. (9) after several iterations, as shown in Fig. 7.

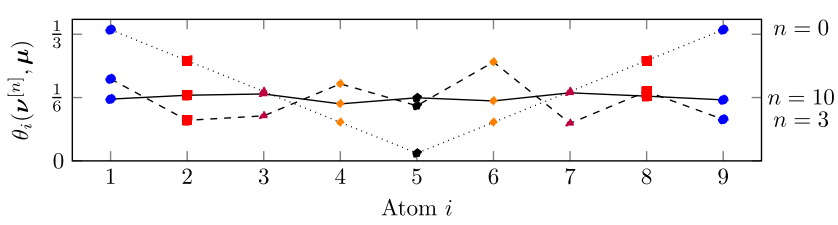

FIG. 7 (color online). The excitation probabilities $\theta_{i}\left(\nu^{[n]}, \boldsymbol{\mu}\right)$ after iterations $n=0,3$, and 10 . 
By manipulating excitation probabilities, we control the populations of mixed states. This can be of interest to (for example) mixed state quantum computing, which lies in between classical computing and quantum computing based on pure, entangled states [31,32]. Creating mixed states can also be a first step towards efficient preparation of large qubit entangled states.

In conclusion, we studied the relations between a physical model of ultracold Rydberg atoms and a stochastic process that models certain wireless random-access networks. This allowed us to identify interesting connections between research fields in physics and mathematics, and to transfer techniques and insights to the realm of Rydberg gases. Our approach can be applied to many other particle systems and stochastic processes as well. Furthermore, the algorithm can be applied to a much larger class of productform networks, with different adjustable parameters [28,33]. Whenever dynamical systems are well described using rate equations, it can be worthwhile to explore possible relations with stochastic processes and cross-pollinate ideas.

This research was financially supported by an ERC Starting Grant, as well as The Netherlands Organization for Scientific Research (NWO), and is part of the research program of the Foundation for Fundamental Research on Matter (FOM). The authors are grateful for the support from Sem Borst and Johan van Leeuwaarden.

*jaron.sanders@tue.nl

Present address: Max Planck Institute for the Physics of Complex Systems, 01187 Dresden, Germany.

[1] R. J. Glauber, J. Math. Phys. (N.Y.) 4, 294 (1963).

[2] T. M. Liggett, Proc. Natl. Acad. Sci. U.S.A. 107, 16413 (2010).

[3] T. M. Liggett, Interacting Particle Systems (Springer, New York, 1985).

[4] T. F. Gallagher, Rydberg Atoms (Cambridge Monographs on Atomic, Molecular and Chemical Physics) (Cambridge University Press, Cambridge, England, 1994).

[5] F. P. Kelly, J. Roy. Stat. Soc. B. 47, 379 (1985).

[6] M. D. Lukin, M. Fleischhauer, R. Cote, L. M. Duan, D. Jaksch, J. I. Cirac, and P. Zoller, Phys. Rev. Lett. 87, 037901 (2001).

[7] D. Comparat and P. Pillet, J. Opt. Soc. Am. B 27, A208 (2010).

[8] D. Jaksch, J. I. Cirac, P. Zoller, S. L. Rolston, R. Côté, and M. D. Lukin, Phys. Rev. Lett. 85, 2208 (2000).

[9] M. Saffman, T. G. Walker, and K. Mølmer, Rev. Mod. Phys. 82, 2313 (2010).

[10] H. Weimer, R. Löw, T. Pfau, and H. P. Büchler, Phys. Rev. Lett. 101, 250601 (2008).

[11] P. Schauss, M. Cheneau, M. Endres, T. Fukuhara, S. Hild, A. Omran, T. Pohl, C. Gross, S. Kuhr, and I. Bloch, Nature (London) 491, 87 (2012).

[12] T. Pohl, E. Demler, and M. D. Lukin, Phys. Rev. Lett. 104, 043002 (2010).
[13] J. Schachenmayer, I. Lesanovsky, A. Micheli, and A. J. Daley, New J. Phys. 12, 103044 (2010).

[14] R. M. W. van Bijnen, S. Smit, K. A. H. van Leeuwen, E. J. D. Vredenbregt, and S. J. J. M. F. Kokkelmans, J. Phys. B 44, 184008 (2011).

[15] M. Höning, D. Muth, D. Petrosyan, and M. Fleischhauer, Phys. Rev. A 87, 023401 (2013).

[16] L. Kleinrock and F. A. Tobagi, IEEE Trans. Commun. 23, 1400 (1975).

[17] J. Sanders, Master's thesis, Eindhoven University of Technology, 2011.

[18] R. R. Boorstyn, A. Kershenbaum, B. Maglaris, and V. Sahin, IEEE Trans. Commun. 35, 267 (1987).

[19] A. Kershenbaum, R. R. Boorstyn, and M. S. Chen, IEEE J. Sel. Areas Commun. 5, 1003 (1987).

[20] C. Ates, T. Pohl, T. Pattard, and J. M. Rost, Phys. Rev. A 76, 013413 (2007).

[21] G. Grimmett and D. Stirzaker, Probability and Random Processes (Oxford University Press, New York, 2001).

[22] F. Robicheaux and J. V. Hernández, Phys. Rev. A 72, 063403 (2005).

[23] F. P. Kelly, Reversibility and Stochastic Networks (Cambridge University Press, Cambridge, England, 2011).

[24] D. A. Levin, Y. Peres, and E. L. Wilmer, Markov Chains and Mixing Times (American Mathematical Society, Providence, 2009).

[25] A. Hu, T. E. Lee, and C. W. Clark, Phys. Rev. A 88, 053627 (2013)

[26] D. Petrosyan, Phys. Rev. A 88, 043431 (2013).

[27] L. Jiang and J. Walrand, in Proceedings of the 46th Annual Allerton Conference on Communication, Control, and Computing, Urbana-Champaign, IL, 2008 (IEEE, New York, 2008).

[28] J. Sanders, S. C. Borst, and J. S. H. van Leeuwaarden, in 6th International Conference on Performance Evaluation Methodologies and Tools (VALUETOOLS), Cargèse, 2012 (IEEE, New York, 2012).

[29] See Supplemental Material at http://link.aps.org/ supplemental/10.1103/PhysRevLett.112.163001 for a brief discussion of the wireless network algorithm that was transferred to the Rydberg gas setting. It explains that the algorithm was designed for controlling the throughput of transmitters, that the algorithm can be implemented in a distributed fashion (i.e., transmitters decide themselves how to change their activity based on local information), and how the algorithm circumvents NP-hard calculations by measuring the throughput of transmitters, instead of calculating the throughput.

[30] P. M. van de Ven, J. S. H. van Leeuwaarden, D. Denteneer, and A. J.E. M. Janssen, Performance Evaluation 69, 121 (2012).

[31] M. Siomau and S. Fritzsche, Eur. Phys. J. D 62, 449 (2011).

[32] R. Blume-Kohout, C. M. Caves, and I. H. Deutsch, Found. Phys. 32, 1641 (2002).

[33] J. Sanders, S. C. Borst, and J. S. H. van Leeuwaarden, in Proceedings of the 50th Annual Allerton Conference on Communication, Control, and Computing (ALLERTON), Monticello, IL, 2012 (IEEE, New York, 2012). 